\title{
Magnetic Flux Determination in Late-Type Dwarfs
}

\author{
P. Rípodas ${ }^{1}$, J. Sánchez Almeida ${ }^{1}$, R. J. García López ${ }^{1}$, \\ M. Collados ${ }^{1,2}$ \\ ${ }^{1}$ Instituto de Astrofísica de Canarias \\ ${ }^{2}$ Departamento de Astrofísica, Universidad de La Laguna \\ E-38200 La Laguna, Tenerife, Spain
}

\begin{abstract}
We present a very preliminary and simplified analysis designed to measure photospheric magnetic fields in late-type stars, using the FeI 5247.06 $\AA$ and $5250.22 \AA$ lines. We show how the use of the equivalent widths of the lines and differences in their depth can give a rapid estimation of the magnetic flux.
\end{abstract}

\section{Introduction}

It is thought that rotationally-induced dynamo mechanisms are responsible for the chromospheric activity observed in late-type stars. One of the reasons for measuring photospheric magnetic fields in stars is to provide an observational basis to test this hypothesis. Confrontation of predictions of photospheric magnetic fluxes produced by dynamo models with observations should then set bounds to the basic parameters of the theory (see Montesinos et al., 1990).

Apart from the uncertainties of the current methods of determining photospheric magnetic fields, the small size of the set of measurements (see Saar, 1990) still restricts the usefulness of this confrontation. We have derived a simple equation that relates the magnetic flux and the equivalent widths and depths of the FeI $5247.06 \AA$ and $5250.22 \AA$ lines. The determination of these parameters from a spectrum is straightforward, so that the relationship may help to enlarge the set of magnetic field strength measurements. 


\section{Determination of the magnetic flux}

A very simple approach for the determination of the magnetic field strength can be expressed as follows:

Assuming a two-component model, the observed flux of a star, normalized to its adjacent continuum, $F(\lambda)$, can be written, in terms of the normalized fluxes of the magnetic and non-magnetic areas, $F_{\mathrm{m}}(\lambda)$ and $F_{\mathrm{nm}}(\lambda)$, as

$$
F(\lambda)=f_{\text {eff }} F_{\mathbf{m}}(\lambda)+\left(1-f_{\text {eff }}\right) F_{\mathrm{nm}}(\lambda) .
$$

$\lambda$ is the wavelength and $f_{\text {eff }}$ represents an effective filling factor (fraction of the surface covered by magnetic regions) given by the expression

$$
f_{\text {eff }}=\frac{f r}{1+(r-1) f}
$$

where $r$ is the continuum contrast between magnetic and non-magnetic areas, and $f$ the actual filling factor.

By observing two lines, very similar in their thermodynamic parameters and with different Landé factors, such as FeI $5247 \AA$ and $5250 \AA$, recentering them and evaluating their difference, one has

$$
\Delta F(\lambda)-\Delta F_{\mathrm{nm}}(\lambda) \approx f_{\mathrm{eff}} \Delta F_{\mathrm{m}}(\lambda) .
$$

This means that, if the observed difference in residual flux between the two lines $(\Delta F(\lambda))$ is larger than the expected value in the absence of a magnetic field $\left(\Delta F_{\mathrm{nm}}(\lambda)\right)$, that difference can be directly attributed to the presence of a magnetic field on the stellar surface.

In order to estimate the magnetic flux, one can make use of eq. (3), and evaluate it at $\lambda=0$ (the center of the lines used). Here we present a very simple analysis, which will be extended in the future to more realistic cases. Thus, assuming that the thermodynamic properties of the magnetic and quiet regions are the same (in which case, $f=f_{\text {eff }}$, among other implications), that the field is longitudinal and weak (Zeeman shift much smaller than the typical width of the lines) and gaussian line profiles (which is valid if the lines are not very strong), one has, for each line:

$$
F_{\mathrm{m}}(0)=1-\frac{W}{\sqrt{2 \pi} \sigma} \exp \left[-\frac{\Delta \lambda^{2}}{2 \sigma^{2}}\right] \approx 1-\frac{W}{\sqrt{2 \pi} \sigma}\left[1-\frac{\Delta \lambda^{2}}{2 \sigma^{2}}\right],
$$

where $\Delta \lambda$ represents the Zeeman splitting, $W$ the equivalent width of the lines (both lines are assumed to have the same $W$, which is true up to an accuracy of $1-2 \%)$, and $\sigma$ is related to the half-width of the lines. By evaluating the difference of FeI $5247 \AA$ and $5250 \AA$, using eq. (4), one has

$$
\Delta F_{\mathrm{m}}(0)=\frac{5 k^{2} \lambda_{0}^{4} B^{2}}{2 \sqrt{2 \pi} \sigma^{3}} W
$$


where $k=4.67 \cdot 10^{-10} \mathrm{~m} \AA$ Gauss $^{-1} \AA^{-2} . \lambda_{0}$ is the central wavelength of one of the lines. Moreover, one can write for the residual flux of FeI $5247 \AA$ (less sensitive to the magnetic field than FeI $5250 \AA$ ),

$$
F_{\mathrm{m}}^{47}(0) \approx 1-\frac{W}{\sqrt{2 \pi} \sigma}\left[1-\frac{\Delta \lambda^{2}}{2 \sigma^{2}}\right] \approx 1-\frac{W}{\sqrt{2 \pi} \sigma}=F_{\mathrm{nm}}^{47}(0)
$$

Combining eqs. (1), (5) and (6), one has an expression for the magnetic flux,

$$
\sqrt{f} B=C W \frac{\left[\Delta F(0)-\Delta F_{\mathrm{nm}}(0)\right]^{1 / 2}}{\left[1-F^{47}(0)\right]^{3 / 2}}
$$

where $C$ is a constant whose value is $\left(\sqrt{5 \pi} k \lambda_{0}^{2}\right)^{-1}=19.6$ Gauss $\AA^{-1}, \Delta F(0)$ is the observed difference between the residual fluxes of the two lines, and $F^{47}(0)$ is the observed residual flux of FeI $5247 \AA$ (although FeI $5250 \AA$ could also be used because of the small filling factors). All these parameters can be directly determined from observed spectra. On the other hand, $\Delta F_{\mathrm{nm}}(0)$ gives account of the intrinsic difference between the two lines, in the absence of magnetic field, and can be calculated using a radiative transfer code.

Although this is a very preliminary analysis, many of the hypotheses can be eliminated with numerical calculations. Different field topologies, integration over the stellar disk, and more realistic spectral line profiles (obtained by integration of the transfer equation) can be introduced to test the validity of eq. (7), or, instead, derive a numerical relationship between the observed parameters which might allow the calculation of the magnetic flux.

As part of an observational programme to measure stellar magnetic fields, we have applied this simple treatment to three late-type dwarfs: $\xi$ Boo A (G8V), $\lambda$ Ser (G0V), and $\beta$ Com (G1V), and have detected magnetic signals in the first two (Ripodas et al., 1990).

\section{References}

Montesinos, B., Fernández Villacañas, J.L., Jordan, C.: 1991, these Proceedings

Rípodas, P., Sánchez Almeida, J., García López, R.J., Collados, M.: 1990, in Surface Inhomogeneities on Late-Type Stars, Armagh, N. Ireland.

Saar, S.H.: 1990, in The Solar Photosphere, ed. J. O. Stenflo, I.A.U. Symp. 132, Kluwer Academic Publishers, Dordrecht, p. 427 